ISSN 0103-8478

\title{
Teores de carboidratos e fluorescência da clorofila $a$ em folhas de limeiras ácidas 'Tahiti' submetidas ao anelamento e incisão anelar de ramos
}

\author{
Levels of carbohydrates and chlorophyll fluorescence $a$ leaves of acid lime trees 'Tahiti' subjected \\ to girdling and ringing of branches
}

\author{
Dierlei dos Santos ${ }^{I^{*}}$ Dalmo Lopes de Siqueira ${ }^{\mathrm{I}}$ Luiz Carlos Chamhum Salomão \\ Paulo Roberto Cecon' ${ }^{I I}$ Girlaine Pereira Oliveira ${ }^{I}$ Daniel Lucas Magalhães MachadoI \\ Moises Zucoloto ${ }^{\mathrm{III}}$
}

RESUMO

Objetivou-se estudar a dinâmica dos carboidratos e a fluorescencia da clorofila a em folhas de limeiras ácidas 'Tahiti', submetidas a épocas diferentes de anelamento e incisão anelar de ramos. Foram estudadas quatro épocas de realização de anelamento e incisão anelar, com um tratamento adicional, sem interrupção do floema. O anelamento, independentemente da época de realização, aumentou os teores de amido (55 a 90\%) e açúcares solúveis totais ( 8 a 18\%) nas folhas do 'Tahiti' até por volta dos 50 aos 60 dias. A incisão anelar, independentemente da época de realização, aumentou a concentração de amido (10 a 35\%) em relação à testemunha, porém, o aumento foi inferior ao que ocorreu nas plantas aneladas (55 a 90\%). O anelamento promoveu aumentos de $F_{0}$ e redução da relação $F / F$, sendo os valores máximos (350) e mínimos (0,78), respectivamente, atingidos por volta dos 60 dias. Para as plantas da testemunha e incindidas, houve aumento de $F_{0}$ sendo o valor máximo atingido aos 50 dias (320), porém inferior aos observados para o anelamento, e não houve alteração da relação $F_{v} / F_{m}$.

Palavras-chave: Citrus latifolia Tan., amido, AST, $F_{0}, F_{v} / F_{m}$

\section{ABSTRACT}

The aimed to study the dynamics of carbohydrates and chlorophyll fluorescence a in leaves of acid lime trees 'Tahiti' subjected to different times girdling and ringing of branches. It was studied four times the realization of girdling and ringing, with an additional treatment, without phloem interruption. The girdling, regardless of the time of realization, increased starch (55 to $90 \%$ ) and soluble sugars levels ( 8 to 18\%) in 'Tahiti' leaves until around 50 to 60 days. The ringing, regardless of achievement time, increased starch content (10 to 35\%) to the control compared, but increase was less than to that which occurred in girdled plants (55 to $90 \%)$. The girdling promoted $F_{0}$ increase and $F_{v} / F_{m}$ ratio reduced, being the maximum (350) and minimum (0.78), respectively, were reached at about 60 days. For control and ringing plants, there was $F_{0}$ increase, being the maximum value was achieved in 50 days (320), but lower than those observed for girdling, and there was no change in Fv/Fm ratio.

Key words: Citrus latifolia Tan., starch, AST, $F_{0}, F_{v} / F_{m}$.

\section{INTRODUÇÃO}

O anelamento e a incisão anelar de ramos são práticas que podem ser usadas para o aumento da produção e fixação de frutos cítricos. Ambos interrompem o transporte via floema, favorecendo o acúmulo de carboidratos nas folhas e ramos acima da região anelada ou incindida, enquanto os frutos (drenos) os reduzem (SARTORI \& ILHA, 2005; RIVAS et al., 2006).

$\mathrm{Na}$ citricultura, o anelamento de ramos traz resultados positivos, principalmente por influenciar o crescimento do fruto e o comportamento reprodutivo da maioria das espécies do gênero Citrus. $\mathrm{O}$ anelamento é efetivo em aumentar a floração, melhorar a frutificação e aumentar o tamanho dos frutos (GOREN et al., 2003).

No que diz respeito ao uso contínuo de anelamento ou incisão anelar em pomares comerciais, acredita-se que, quando feitos de forma adequada (espessura do anel, sanidade, nutrição e frequência da

\footnotetext{
IDepartamento de Fitotecnia, Universidade Federal de Viçosa (UFV), Av. PH Rolfs, s/n, 36570-000, Viçosa, MG, Brasil. E-mail: dierlei@ vicosa.ufv.br.*Autor para correspondência.

IIDepartamento de Estatística, UFV, Viçosa, MG, Brasil.

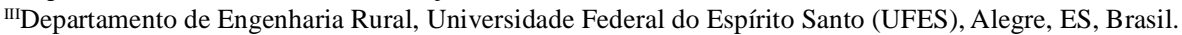
Recebido 05.10.13 Aprovado 26.02.14 Devolvido pelo autor 13.07.14 CR-2013-1316.R3
} 
operação compatível com o porte da planta), podem ser repetidos durante vários anos, sem que sejam prejudiciais às plantas (GUARDIOLA, 1992).

Apesar dos benefícios mencionados do anelamento, em alguns casos, pode induzir acúmulo excessivo de carboidratos, dando origem a uma retroinibição da fotossíntese, reduzindo a capacidade fotossintética (RIVAS et al., 2007) e a assimilação de $\mathrm{CO}_{2}$ (IGLESIAS et al., 2003). Redução na fotossíntese devido ao uso da prática de anelamento foi relatada em outros citros como Citrus unshiu [Mak.] Marc.) cv. 'Okitsu' (IGLESIAS et al., 2006), Citrus reticulata Hort. ex Tan. x Citrus paradisi Macf. x C. tangerina Hort. ex Tan ('Nova') e Citrus reticulata Blanco ('Loretina') (RIVAS et al., 2007; 2008; 2011)

Outros autores relatam que o acúmulo de carboidratos em folhas, durante o período diurno, pode comprometer a taxa de transporte de elétrons e causar danos a membranas dos tilacoides, causando desarranjo e interrupção do sistema de membrana interna, fenômeno conhecido com estresse oxidativo (BONDADA \& SYVERTSEN, 2005).

Talvez o efeito prejudicial mais visível do anelamento seja o amarelecimento das folhas e o retardamento do crescimento vegetativo das plantas de 'Tahiti'. PEREIRA et al. (2010) constataram redução no índice Spad em plantas aneladas (redução da cor verde) e relacionaram esse efeito à redução da concentração nutrientes na parte aérea da planta.

Em condições ambientais estressantes, ocorre decréscimo na eficiência quântica potencial do fotossistema II (PSII), que pode ser detectada pela queda da eficiência fotoquímica máxima do PSII, ou seja, decréscimo da fluorescência variável em relação à fluorescência máxima $\left(F / F_{m}\right)$ (KRAUSE \& WEIS, 1991). A relação $F_{v} / F_{m}$ representa a eficiência máxima que a luz absorvida pela antena do PSII é convertida em energia química (BAKER, 2008). Plantas de citros (Híbridos 'Fortuna' e 'Nova', Clementina 'Loretina' e Satsuma 'Clauselina') e manga (Cv. 'Cogshall') submetidas ao anelamento apresentaram folhas com eficiência quântica do PSII (ФPSII) reduzida (RIVAS et al., 2006; 2007; 2008).

Apesar de todas essas constatações, não foram encontrados, na literatura, trabalhos comparativos, relacionados aos aspectos fisiológicos entre o uso da incisão anelar e anelamento em limeira ácida 'Tahiti'. Por isso, objetivou-se estudar a dinâmica dos carboidratos e fluorescência da clorofila $a$ em folhas de limeiras ácidas 'Tahiti' submetidas a épocas diferentes de anelamento e incisão anelar nos ramos.

\section{MATERIAL E MÉTODOS}

O experimento foi desenvolvido em propriedade particular localizada na latitude $20^{\circ} 46^{\prime} 20^{\prime \prime}$ Sul e na longitude $43^{\circ} 04^{\prime} 04^{\prime \prime}$ Oeste, no município de Viçosa - MG. Utilizaram-se limeiras ácidas 'Tahiti' clone IAC-5 enxertadas sobre limoeiro 'Cravo', plantadas no espaçamento de 4x3m, irrigadas por microaspersão e com idade de quatro anos. As plantas encontravam-se uniformes quanto ao porte, produção, sanidade e estado nutricional.

$\mathrm{O}$ delineamento usado foi o de blocos ao acaso, com duas plantas por parcela, em esquema fatorial, composto por quatro épocas de realização e duas formas de interrupção do floema, com um tratamento adicional, sem interrupção do floema. Foram realizadas avaliações ao longo do tempo, após a aplicação dos tratamentos até a colheita dos frutos. As duas formas de interrupção do floema foram o anelamento e incisão anelar e as datas de aplicação desses tratamentos (T) foram as seguintes: T1: Testemunha (sem incisão e anelamento); Anelamento - T2 (15/06/10), T4 (05/07/10), T6 (25/07/10) e T8 (14/08/10); Incisão anelar - T3 (15/06/10), T5 (05/07/10), T7 $(25 / 07 / 10)$ e T9 (14/08/10). O pleno florescimento ocorreu na semana do dia 02/09, cerca de 20 dias após a realização do último tratamento.

$\mathrm{O}$ anelamento foi realizado em todos os ramos principais das plantas, $10 \mathrm{~cm}$ acima do ponto de ramificação do tronco, rompendo o floema em toda a sua circunferência, sem, no entanto, danificar o xilema. Foi realizado com um canivete de lâmina dupla que permitiu a retirada de um anel de casca de exatamente dois $\mathrm{mm}$ de espessura. A incisão anelar consistiu de um corte completo realizado com canivete de enxertia no córtex dos ramos principais, $10 \mathrm{~cm}$ acima do ponto de emissão das pernadas, sem afetar o xilema e sem a retirada da casca.

Os parâmetros de fluorescência da clorofila foram medidos usando fluorômetro ADC FIM 1500 (ADC Bioscientific Ltd, Hodderston, UK) (fluorescência direta). Foram avaliadas a fluorescência inicial $\left(\mathrm{F}_{0}\right)$ e eficiência fotoquímica máxima do PSII, dada pela relação da fluorescência variável pela fluorescência máxima $\left(F_{v} / F_{m}\right)$. As leituras foram realizadas entre as $7 \mathrm{~h}$ e $11 \mathrm{~h}$, em uma folha por planta, utilizando-se sempre a quarta folha madura a partir do ápice, de ramos localizados na região mediana e externa da planta, no quadrante que recebia o sol nascente. Essas avaliações foram realizadas aos 0, 20, 40 dias após a aplicação dos tratamentos e, a partir daí, a cada 30 dias, até a colheita dos frutos. 
Foram avaliados os teores foliares de açúcares solúveis totais e amido, cujas amostras foram colhidas aos 0, 20, 40 dias após a aplicação dos tratamentos e, a partir daí, a cada 30 dias. Foram coletadas duas folhas maduras ( $3^{\mathrm{a}} \mathrm{e} 4^{\mathrm{a}}$ folhas contadas a partir do ápice do ramo), completamente expandidas, de cada quadrante, totalizando oito folhas por planta. Estas, foram lavadas com detergente não-iônico, enxaguadas com água destilada por três vezes e secas em papel de filtro. Em seguida, foram secas em estufa de circulação forçada de ar, a $60^{\circ} \mathrm{C}$, até obtenção de peso constante.

Posteriormente, as amostras foram moídas em moinho tipo Willey, de aço inoxidável, passadas em peneira de 20 mesh, e acondicionadas em frascos para posterior análise química. A obtenção dos extratos e a determinação das concentrações do amido e açúcares solúveis totais foram realizadas de acordo com o método descrito por McCREADY et al. (1950).

Os dados foram submetidos à análise de regressão e os modelos escolhidos com base na significância dos coeficientes ao nível de $10 \%$ de probabilidade pelo teste " $\mathrm{t}$ ", no coeficiente de determinação $\left(\mathrm{R}^{2}=\mathrm{SQ}_{\mathrm{Reg}} / \mathrm{SQ}_{\text {Trat }}-\mathrm{SQ}_{\mathrm{Reg}}=\right.$ soma de quadrado da regressão; $\mathrm{SQ}_{\text {Trat }}=$ soma de quadrado de tratamento) e no potencial para explicar o fenômeno em estudo.

\section{RESULTADOS E DISCUSSÃO}

O anelamento, independentemente da época em que foi realizado, alterou significativamente os teores foliares de amido (Figura 1). Os maiores teores foram observados por volta dos 50 aos 60 dias após a realização do anelamento, retornando a sua normalidade por volta dos 120 dias, provavelmente devido ao restabelecimento do transporte no floema, dado que a atividade do câmbio produz calos e conexões vasculares que restabelecem o fluxo normal (WILLIAMS et al., 2000).

O aumento nos teores de carboidratos ocorre porque o anelamento ou a incisão bloqueiam

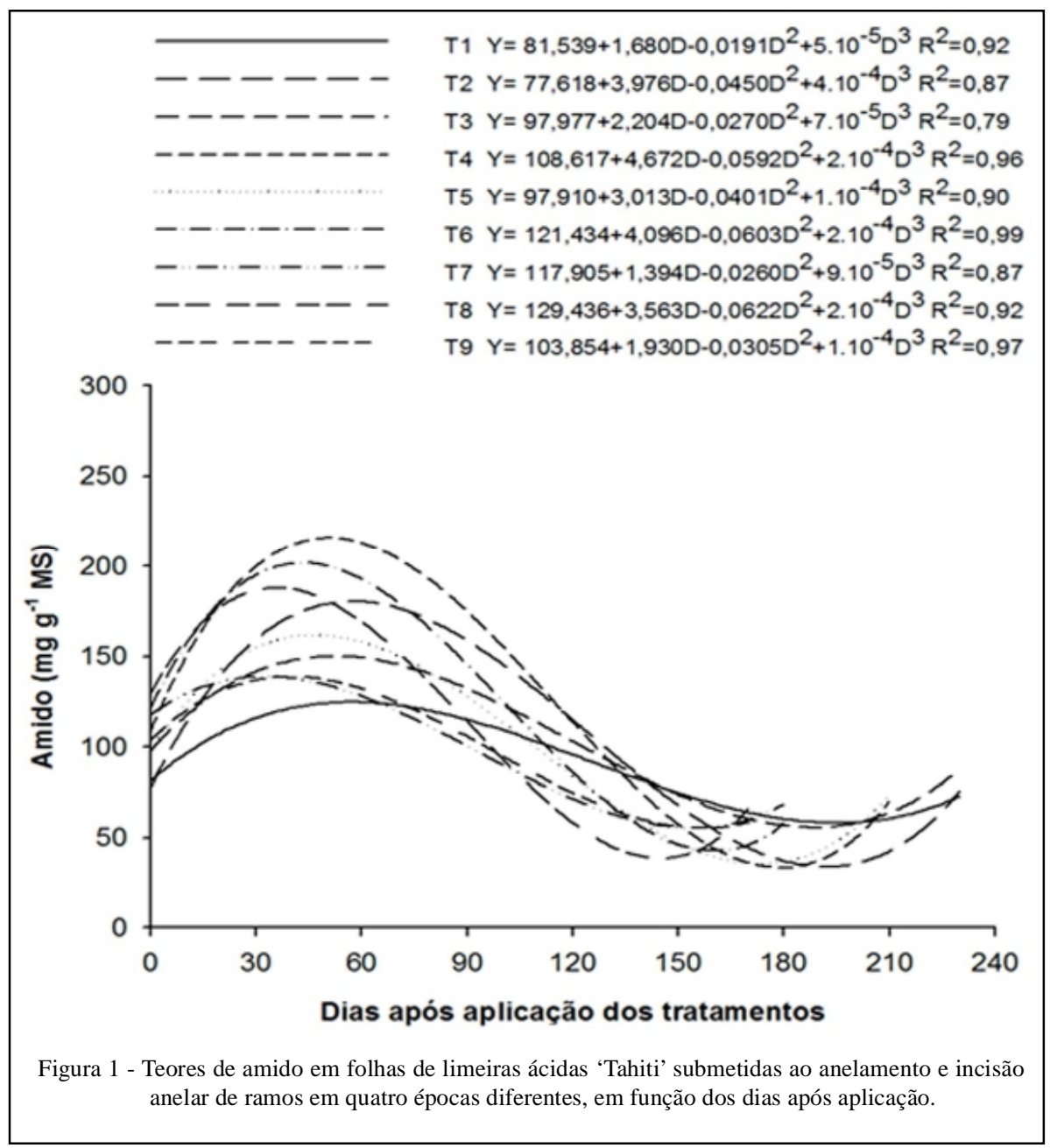

Ciência Rural, v.44, n.10, out, 2014. 
o transporte de fotoassimilados da parte aérea para as raízes, resultando em incrementos dos teores nas folhas. Desse modo, os carboidratos tornam-se disponíveis para o estabelecimento e a produção de frutos (RIVAS et al., 2006; 2007; 2008; MAHOUACHI et al., 2009).

Em relação à incisão anelar, poucas foram as alterações causadas por essa prática nesse experimento, sendo a principal o aumento da concentração de amido. Segundo SARTORI \& ILHA (2005), seu efeito em fruteiras de caroço, bloqueando o transporte de fotoassimilados, permanece durante aproximadamente 10 a 15 dias. Para citros, segundo COHEN (1981), um anelamento de $2-3 \mathrm{~mm}$ de espessura se restabelece depois de 40-85 dias, enquanto que a incisão anelar se restabelece em um prazo de 7-15 dias (LEWIS \& McCARTY, 1973).

Houve incremento nas concentrações de açúcares solúveis totais (AST) a partir da aplicação dos tratamentos, sendo que as maiores concentrações ocorreram entre 30 e 60 dias após, mais expressivas nas plantas que receberam anelamento, independentemente da época de realização (Figura 2).

Anelamentos geralmente resultam em aumentos significativos nas concentrações de AST e amido em um prazo que varia de 10 a 70 dias, porém, as concentrações de AST variam menos do que as de amido (URBAN \& ALPHONSOUT, 2007; RIVAS et al., 2008). Uma das explicações para justificar o baixo acúmulo de AST pode ser que estão servindo de esqueletos carbonados para a síntese de numerosos compostos usados para o crescimento das plantas (TAIZ \& ZEIGER, 2009).

A Fluorescência inicial $\left(\mathrm{F}_{0}\right)$ representa a emissão de luz pelas moléculas de clorofilas $a$ excitadas, antes de a energia ser dissipada para o centro de reação do PSII, sendo independente dos eventos fotoquímicos. É um valor referência para a determinação das outras variáveis da fluorescência (HIPKINS \& BAKER, 1986).

$\mathrm{O}$ valor de $\mathrm{F}_{0}$ pode ser modificado por quaisquer estresses que causam alterações estruturais nos pigmentos fotossintéticos do PSII (BAKER, 2008), nesse caso, promovidos pelo

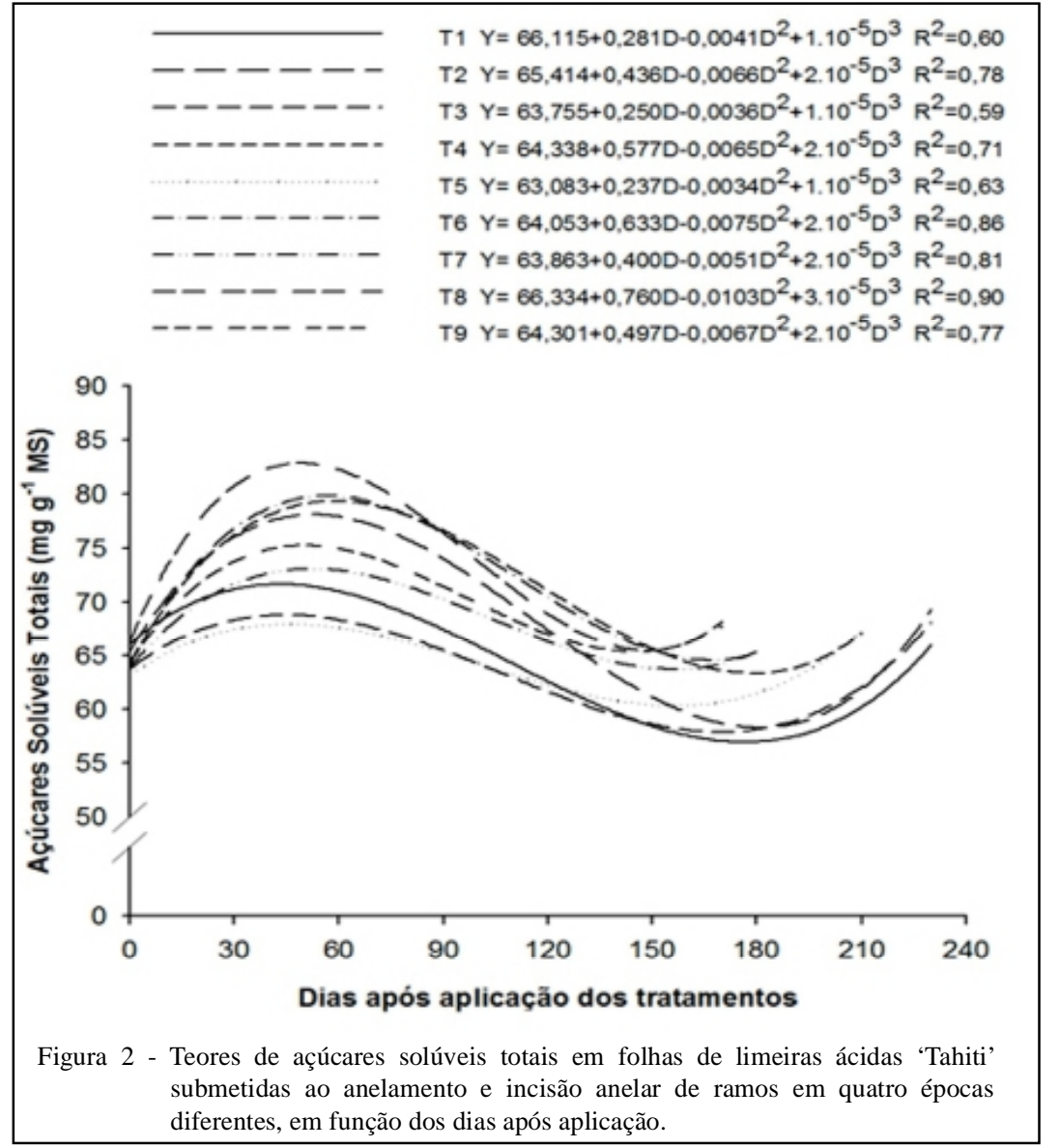

Ciência Rural, v.44, n.10, out, 2014. 
acúmulo de amido nas plantas submetidas ao anelamento.

Houve pequeno acréscimo dos valores de $\mathrm{F}_{0}$ nas folhas das plantas incindidas e aneladas, após aplicação dos tratamentos, sendo o máximo atingido por volta dos 50 dias (Figura 3).

Para o caso em estudo, todas essas alterações estruturais podem ter sido provocadas pelo acúmulo de amido em forma de grânulos nos tilacoides, justificando os aumentos simultâneos ocorridos nas concentrações de amido e nos valores de $\mathrm{F}_{0}$. Esses resultados também são compartilhados com outros autores que trabalharam com citros (BONDADA \& SYVERTSEN, 2005).

As plantas incindidas não apresentaram variação na relação $F_{v} / F_{m}$ ao longo do tempo, semelhantemente à testemunha, e com valores superiores a 0,81 . Por outro lado, houve redução da relação $F / F_{m}$ nas plantas submetidas ao anelamento, partindo de lavores próximos a 0,82 no início do experimento, e atingindo valores mínimos $(0,78)$ por volta dos 50-70 dias, e retornando a serem semelhantes à testemunha aos 160-180 dias (Figura 4).

A relação $F_{v} / F_{m}$ representa a eficiência máxima que a luz absorvida pela antena do PSII é convertida em energia química (em condições adequadas, apresenta valores em torno de 0,80 ) e é um indicador da ocorrência de fotoinibição, quando plantas estão sujeitas a vários tipos de estresses (BAKER, 2008). Quando a disponibilidade de energia de excitação é superior à capacidade de utilização na fotossíntese (reações bioquímicas), há fotoinibição, indicada por valores de $\mathrm{F}_{\mathrm{v}} \mathrm{F}_{\mathrm{m}}^{-1}$ menores que 0,725 (CRITCHLEY, 1998). Neste trabalho, os valores da relação $F_{v} / F_{m}$ encontrados, em nenhum momento, foram inferiores a 0,725 , considerado limite inferior para ocorrência de fotoinibição, apesar do acúmulo considerável de carboidratos.

\section{CONCLUSÃO}

$\mathrm{O}$ anelamento de ramos, independente da época de realização, aumentou a concentração

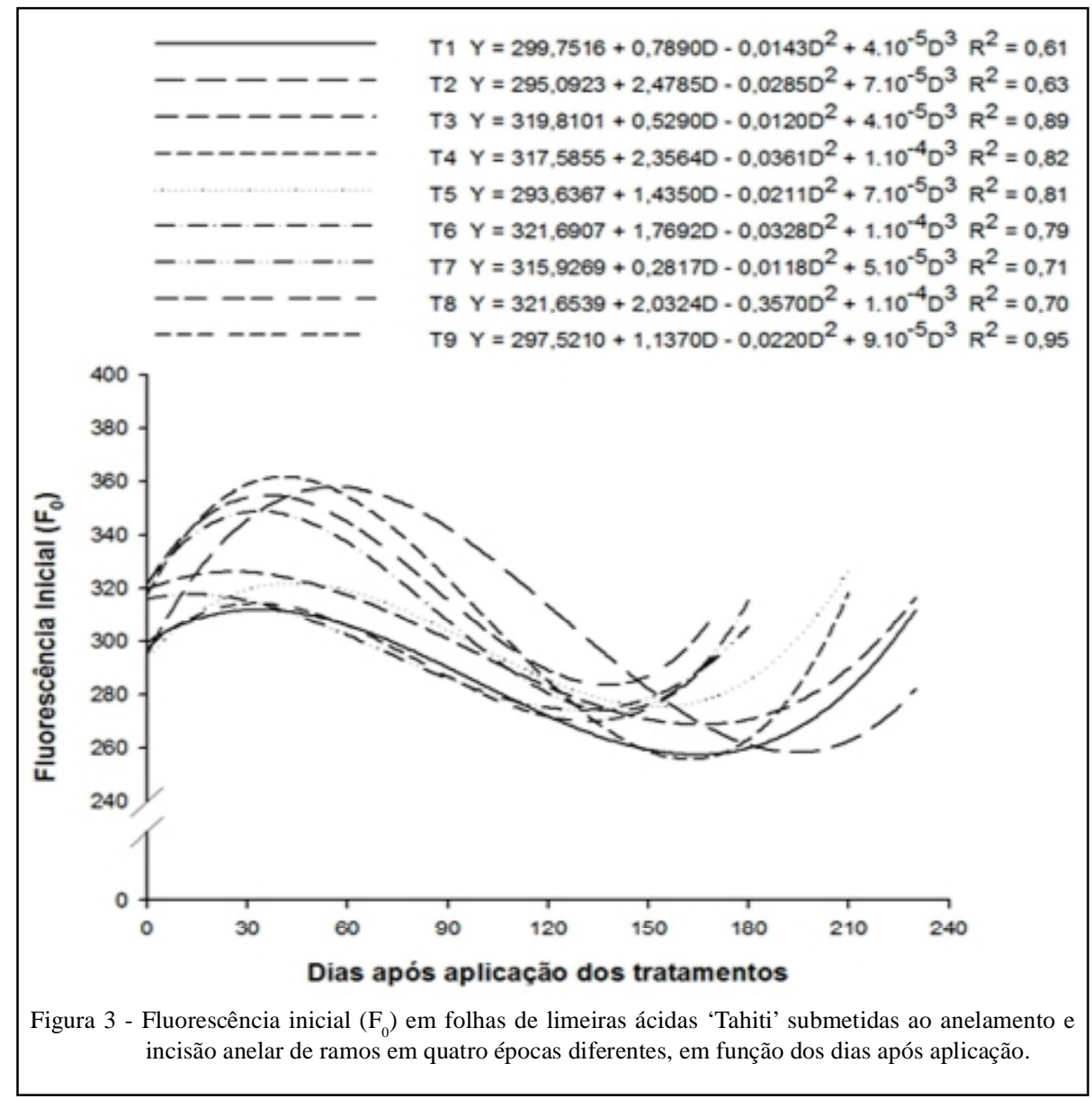

Ciência Rural, v.44, n.10, out, 2014. 


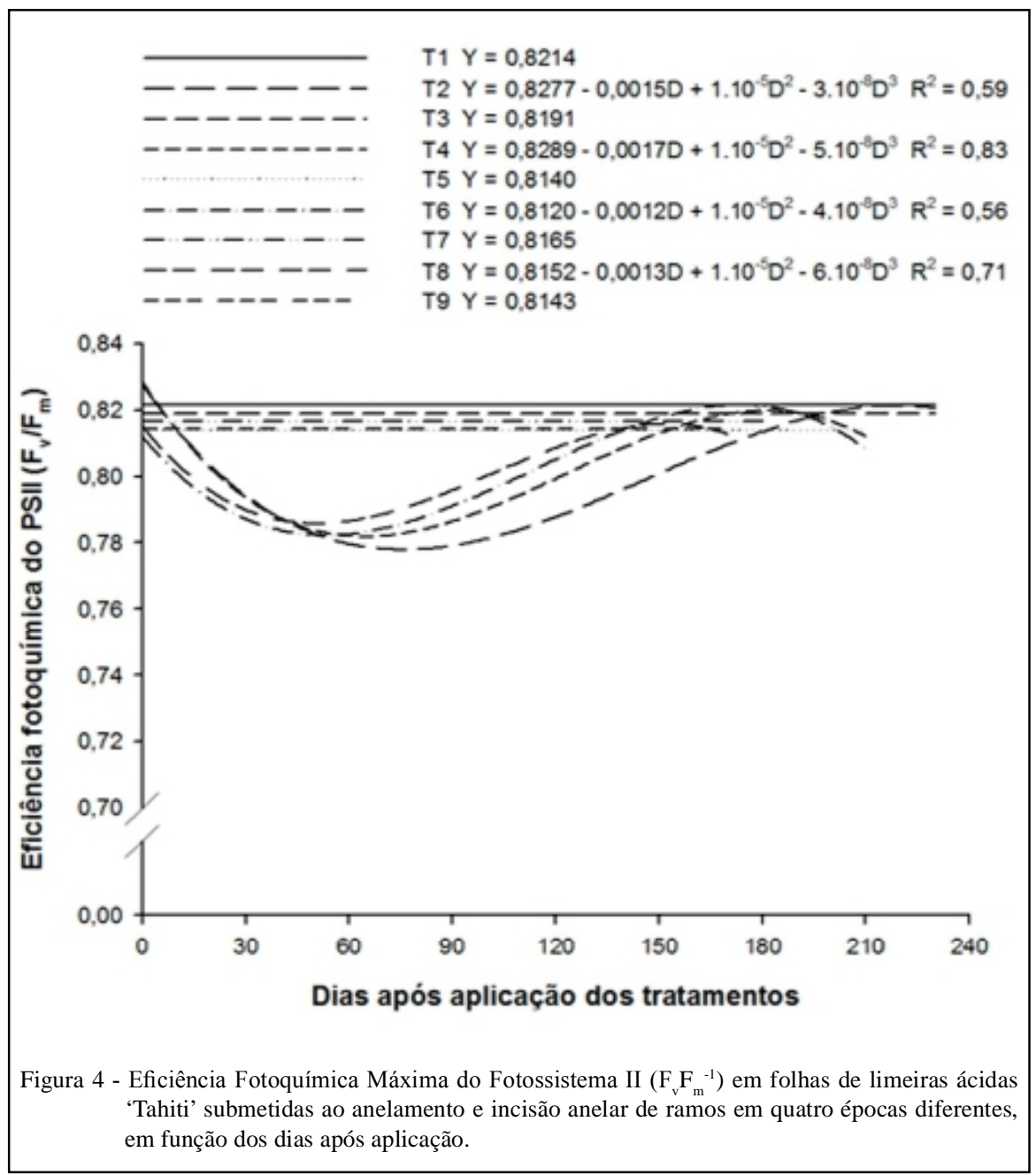

de amido e açúcares solúveis totais em folhas de limeira ácida 'Tahiti'. Tanto o anelamento quanto a incisão não foram capazes de promover fotoinibição da fotossíntese, apesar do acúmulo considerável de carboidratos.

\section{AGRADECIMENTOS}

Ao Conselho Nacional de Desenvolvimento Científico e Tecnológico (CNPq), pela concessão da bolsa de Doutorado ao primeiro autor.

\section{REFERÊNCIAS}

BAKER, N.R. Chlorophyll fluorescence a probe of photosynthesis in vivo. Annual Review of Plant Biology, v.59, p.89-113, 2008. Disponível em: <http://www.annualreviews.org/doi/pdf/10.1146/ annurev.arplant.59.032607.092759>. Acesso em: 18 maio. 2013. doi: 10.1146/annurev.arplant.59.032607.092759.

BONDADA, B.R.; SYVERTSEN, J.P. Concurrent changes in net $\mathrm{CO}_{2}$ Assimilation and chloroplast ultrastructure in nitrogen deficient citrus leaves. Environmental and Experimental Botany, v.54, n.1, p.41-48, 2005. Disponível em: <http://www. crec.ifas.ufl.edu/academics/faculty/syvertsen/PDF/BB_SYV_ EEB_Ac_N_2005.pdf>. Acesso em: 12 jun. 2013. doi: 1016/j. envexpbot.2004.05.005.

COHEN, A. Recent developments in girdling of citrus tree. Proceedings of the International Society of Citriculture, v.1, p.196-199, 1981.

CRITCHLEY, C. Photoinhibition. In: RAGHAVENDRA, A.S. Photosynthesis: a comprehensive treatise. Cambridge: Cambridge University, 1998. Chap.20, p.64-272.

GOREN, R. et al. Girdling: physiological and horticultural aspects. Horticultural Reviews, v.30, p.1-36, 2003. Disponível em: <http:// onlinelibrary.wiley.com/doi/10.1002/9780470650837.ch1/pdf > . Acesso em: 21 ago. 2013. doi: 10.1002/9780470650837.ch1.

GUARDIOLA, J.L. Frutificação e crescimento. In: SEMINÁRIO INTERNACIONAL DE CITROS: FISIOLOGIA, 2., 1992, Bebedouro. Seminário... Bebedouro: Fundação Cargill, 1992. p.1-26. 
HIPKINS, M.F.; BAKER, N.R. In: HIPKINS, M.F.; BAKER, N.R. (Eds.). Photosynthesis-energy transduction: a practical approach. Oxford: IRL, 1986. 51p.

IGLESIAS, D.J. et al. Carbohydrate and ethylene levels related to fruitlet drop through abscission zone A in citrus. Trees, v.20, p.348-355, 2006. Disponível em: <http://download.springer.com/ Static/pdf/69/art\%253A10.1007\%252Fs00468-005-0047-x.pdf?a uth66 $=1380544133$ 066aecd3b3d71c2e3cf31b8ba614082f\&ext= pdf $>$. Acesso em: 12 jun. 2013. doi: 10.1007/s00468-005-0047-x.

IGLESIAS, D.J. et al. Fruit set dependence on carbohydrate availability in citrus trees. Tree Physiology, v.23 p.199204, 2003. Disponível em: <http://treephys.oxfordjournals. org/content/23/3/199.long $>$. Acesso em: 15 jun. 2013. doi: 10.1093/treephys/23.3.199.

KRAUSE, G.H.; WEIS, E. Chlorophyll fluorescenceand photosynthesis: the basics. Annual Review of Plant Physiology and Plant Molecular Biology, v.42, p.313-349, 1991. Disponível em: <http://www. annualreviews.org/doi/pdf/10.1146/annurev.pp.42.060191.001525>. Acesso em: 17 ago. 2013. doi: 1040-2519/91/0601-0313\$02.00.

LEWIS, L.N.; McCARTY, C.D. Pruning and girdling of citrus. In: REUTHER, W. (Ed.). The citrus industry. Berkeley: Univ. of California, 1973. V.3. p.211-229.

MAHOUACHI, J. et al. Delay of early fruitlet abscission by branch girdling in citrus coincides with previous increases in carbohydrate and gibberellin concentrations. Plant Growth Regulation, v.58, p.15-23, 2009. Disponível em: <http://download.springer.com/ static/pdf/679/art\%253A10.1007\%252Fs10725-008-9348-.pdf?auth 66=1380389848_14f7ef10d9b72ccf60a30e3f576ab19c\&ext=.pdf $>$. Acesso em: 16 maio, 2013. doi: 10.1007/s10725-008-9348-6.

McCREADY, R.M. et al. Determination of starch and amylase in vegetables. Application to peas. Analytical Chemistry, Washington, v.22, p.1156-1158, 1950. Disponível em: <http:// pubs.acs.org/doi/pdf/10.1021/ac60045a016>. Acesso em: 20 maio, 2013. doi: 10.1021/ac60045a016.

PEREIRA, C.S. et al. Crescimento vegetativo e intensidade de cor verde das folhas de limeira ácida 'Tahiti' anelada e tratada com ácido giberélico. Ciência Rural, v.40, p.1916-1921, 2010. Disponível em: 〈http://www.scielo.br/pdf/cr/v40n9/a707cr3736.pdf〉. Acesso em: 20 maio, 2013. doi: 10.1590/S0103-84782010005000145.
RIVAS, F. et al. Girdling increases carbohydrate availability and fruit-set in citrus cultivars irrespective of parthenocarpic ability. Journal of Horticultural Science and Biotechnology, v.81, p.289-295, 2006. Disponível em: <http://www.crec.ifas. ufl.edu/academics/classes/hos6546/materials/Jou_Hort_Sci_ Biotech_81_2_289-295.pdf>. Acesso em: 29 jun. 2013.

RIVAS, F. et al. Girdling induces oxidative damage and triggers enzymatic and non-enzymatic antioxidative defences in Citrus leaves. Environmental and Experimental Botany, v.64, p.256-263, 2008. Disponível em: <http://dx.doi.org/10.1016/j. envexpbot.2008.07.006>. Acesso em: 16 jun. 2013. doi: 10.1016/j. envexpbot.2008.07.006

RIVAS, F. et al. Changes in carotenoids and ABA content in Citrus leaves in response to girdling. Scientia Horticulturae, v.127, p.482-487, 2011. Disponível em: <http://dx.doi.org/10.1016/j. scienta.2010.11.010>. Acesso em: 27 ago. 2013. doi: 10.1016/j. scienta.2010.11.010.

RIVAS, F. et al. Girdling effects on fruit set and quantum yield efficiency of PSII in two Citrus cultivars. Tree Physiology, v.27, p.527-535, 2007. Disponível em: <http://treephys.oxfordjournals org/content/27/4/527.full.pdf+html>. Acesso em: 22 jun. 2013. doi: $10.1093 /$ treephys/27.4.527.

SARTORI, I.A.; ILHA, L.L.H. Anelamento e incisão anelar em fruteiras de caroço. Ciência Rural, v.35, n.3, p.724-729, 2005. Disponível em: 〈http://www.scielo.br/pdf/cr/v35n3/a40v35n3.pdf>. Acesso em: 27 ago. 2013. doi: 0.1590/S0103-84782005000300040>.

TAIZ, L.; ZEIGER, E. Fisiologia vegetal. 4.ed. Porto Alegre: Artmed, 2009. 819p.

URBAN, L.; ALPHONSOUT, L. Girdling decreases photosynthetic electron fluxes and induces sustained photoprotection in mango leaves. Tree Physiology, v.27, p.345-352, 2007. Disponível em: <http://treephys.oxfordjournals.org/content/27/3/345.full.pdf+html>. Acesso em: 20 maio, 2013. doi: 10.1093/treephys/27.3.345.

WILLIAMS, L.E. et al. Effect of girdling on leaf gas exchange, water status, and non-structural carbohydrates of field-grown Vitis vinifera L. (cv. 'Flame Seedless'). American Journal of Enology and Viticulture, v.51, p.49-54, 2000. 